\title{
Auditory matching-to-sample in monkeys (Cebus apella)
}

\author{
M. R. D'AMATO and MICHAEL COLOMBO \\ Rutgers-The State University, New Brunswick, New Jersey
}

\begin{abstract}
Four of 8 monkeys were successfully trained on an identity matching-to-sample task employing two acoustic stimuli. In five subsequent tests with different pairs of auditory stimuli, their performances were at levels that provided strong evidence for concept-mediated transfer. Thus, despite past failures to demonstrate the matching concept in the auditory modality, the present results indicate that the matching concept is not limited in monkeys to the visual modality. On the other hand, the failure of 4 subjects to learn the initial matching task constitutes additional evidence of cognitive asymmetry in monkeys with regard to the visual and auditory modalities.
\end{abstract}

Much of our knowledge of monkeys' cognitive capacities is based on the visual modality. This is not entirely accidental, because, despite a rich vocal communication system, monkeys often encounter a great deal of difficulty learning simple or complex tasks when acoustic, rather than visual, stimuli serve as the discriminative stimuli (e.g., Cowey, 1968; D'Amato \& Salmon, 1982; Thompson, 1980; Wegener, 1964). Matching-to-sample is a case in point. Monkeys readily learn to match visual cues, and they transfer to new visual stimuli at a level of performance that constitutes strong evidence for the matching concept in this modality (e.g., D'Amato, Salmon, \& Colombo, 1985). On the other hand, Thompson (1980) was able to train only 1 of 4 rhesus monkeys to match auditory stimuli, and not to a very high level of accuracy at that (only about $75 \%$ correct). Moreover, this animal's performance on a subsequent transfer test suggested that it had learned the original matching task on the basis of specific sample/comparison stimulus configurations; it gave no evidence of having learned a matching rule (Thompson, 1981). Using a variety of shaping techniques, we tried without success to train cebus monkeys to match auditory stimuli. Yet the monkeys matched visual stimuli within the same experimental paradigm with ease (D'Amato \& Salmon, 1984b; Salmon, 1984). A similar problem was encountered by A. A. Wright with a rhesus monkey (personal communication, September 1985).

This apparent asymmetry in the degree to which the visual and auditory modalities support learning and cognitive functions prompted Thompson (1981) to suggest that the complex cognitive capacities of animals might be modality specific. This is to say, different species (for example, monkeys and dolphins) may have evolved similar

This research was supported by NSF Grants 8207146 and 8417383 . Incomplete results from three subjects were briefly described at the XXIII International Congress of Psychology held in 1984, in Acapulco, Mexico (D'Amato \& Colombo, 1985). Reprints may be obtained from M. R. D'Amato, Department of Psychology, Rutgers-The State University, New Brunswick, NJ 08903. cognitive capacities which, in each case, are limited to the species' dominant sensory modality.

Although this hypothesis appears attractive on the basis of the previously cited data, other results suggest that the situation may be more complex. For example, the dolphin, thought to be cognitively impoverished in the visual modality, has been reported to acquire language-like behavior with equal facility when acoustic or visual stimuli served as the discriminative stimuli (Herman, Richards, \& Wolz, 1984). According to these investigators, visual discriminative stimuli pose a problem for dolphins when they are "static" but not when they are "dynamic" (incorporate temporal patterning), as employed in their study.

Another relevant fact is that Stepien and Cordeau (1960) successfully trained African green monkeys to match click rates varying in frequency between 5 and $20 \mathrm{~Hz}$. We have suggested that there may be a general coding process for simple repetition rate that is largely independent of the transmitting sensory modality, which would account for their finding that some monkeys immediately generalized matching of click rate to matching light flicker in the same frequency range (D'Amato \& Salmon, 1984b). If this interpretation were correct, the results of Stepien and Cordeau would not be particularly damaging to the hypothesis of modality specificity of cognitive processes. Still, the very fact that Stepien and Cordeau's monkeys were able to match auditory stimuli, even if only on the basis of repetition rate, raises the possibility that the monkey's sensitivity to acoustic stimuli might be significantly enhanced by favorable training procedures.

The present experiment was addressed to this issue. Employing procedures that experience and reason suggested would increase our monkeys' sensitivity to acoustic stimuli, we once again attempted to obtain auditory matching based on differences in frequency. If we were successful in this objective, our more ambitious aim was to demonstrate the matching concept in the auditory modality. This important question was not explored by Stepien and Cordeau (1960). 


\section{METHOD}

\section{Subjects}

Eight Cebus apella monkeys, 6 males and 2 females, ranging in age between 4 and 22 years, served as subjects. Only 2 of the monkeys (Dagwood and Fifi) were not laboratory born. Their previous experimental experience with visual stimuli varied greatly, and all but 1 subject (Tom) had had previous training on visual matchingto-sample. All but 2 (Fifi and Phurp) had had previous experience with acoustic stimuli within an operant discrimination setting (D'Amato \& Salmon, 1982, 1984a). Throughout the experiment, the animals were given a daily ration of Purina Monkey Chow No. 5045 ( 2 to $3 \mathrm{~h}$ following an experimental session), adjusted to the maximum amount that supported reliable performance in each monkey. The daily rations were sufficient to maintain the animals at $90 \%-100 \%$ of their free-feeding body weights.

\section{Apparatus}

The experimental apparatus, which has been described in detail elsewhere (D'Amato \& Salmon, 1982), was simple in its essentials. The front wall of the experimental chamber accommodated two response levers which were separated by $43 \mathrm{~cm}$, each $22 \mathrm{~cm}$ above the chamber floor. A speaker $(10-\mathrm{W}, 10-\mathrm{cm}, 8-\mathrm{ohm}$ Realistic Model FE-103) was located directly below each lever. The right speaker presented the acoustic sample stimuli, and the left speaker presented the comparison stimuli. Illumination was provided by an overhead houselight, which could be turned on or off or dimmed. Noyes banana-flavored pellets $(190 \mathrm{mg})$, delivered to a food cup located on the right wall of the chamber, served as reinforcers.

The acoustic stimuli were generated by a Commodore PET microcomputer, the signals amplified by the amplifier portion of a MTU D/A converter (Model K-1002). Three tones were employed, either alone or in combination: a $243-\mathrm{Hz}$ low-frequency tone (LT), a $975-\mathrm{Hz}$ medium-frequency tone (MT), and a $4165-\mathrm{Hz}$ high-frequency tone (HT). There was also a high-frequency tune ("gliss"), which consisted of 11 monotonically ascending and 11 monotonically descending frequencies ranging between 1469 and $9613 \mathrm{~Hz}$ (similar to the gliss tune used in D'Amato \& Salmon, 1982). The duration of one complete playing of the gliss tune was about $1.5 \mathrm{sec}$. The intensity of the acoustic stimuli, measured on the $\mathrm{C}$ scale of a Simpson sound-level meter (Model 886) positioned about $30 \mathrm{~cm}$ from the sound source, was $75-80 \mathrm{~dB}$ (re .0002 $\mu$ bar).

Presentation of trials events and data recording were accomplished by a PDP $8 / \mathrm{e}$ minicomputer and a Commodore microcomputer and disk units.

\section{Procedure}

Several measures were taken to facilitate learning of the matching task. Acquisition of a simple auditory discrimination seems to be facilitated when the sound source is close to the manipulandum (D’Amato \& Salmon, 1982; Segal \& Harrison, 1978); as already noted, the speakers delivering the acoustic stimuli were located directly below the response levers. Moreover, to assist differentiation of the samples and comparison stimuli, the sample stimuli were presented by the right speaker only and the comparison stimuli were restricted to the left speaker. In our previous attempts to obtain auditory matching, we tried to shape auditory matching behavior by first training the monkeys on visual matching and then introducing the auditory stimuli within the same general paradigm. Several variations on this theme failed, possibly because the earlier presence of visual discriminative stimuli strongly biased the animals to attend to visual cues, attenuating their sensitivity to acoustic stimuli. Abandoning this strategy, the present study introduced auditory stimuli from the beginning in a go/no-go paradigm which had not been previously used in visual matching. Finally, the pair of stimuli used in the initial matching task consisted of the gliss tune and the (steady) LF tone, which are easily differentiated on the basis of mean frequency, frequency envelope, or tempo.
Training on the initial matching task. The subjects were first shaped to press the right lever to obtain food pellets; they were then trained to press the right and left levers in sequence, a food pellet being delivered after completion of the sequence. Over the next few sessions, the animals were gradually introduced to the format of a matching trial, which was as follows. A trial began with the houselight illuminated; $2 \mathrm{sec}$ later, a sample stimulus was presented by the speaker located under the right response lever. Responses on the right lever were ineffective during the first $2 \mathrm{sec}$ after onset of the sample (an enforced "listening" period). The first response on the right lever after the 2 -sec listening period terminated the sample stimulus, extinguished the houselight, and initiated a brief delay interval $(.5 \mathrm{or} 1.0 \mathrm{sec})$. The point of introducing a delay interval marked by a distinguishing houselight condition was to encourage the monkeys to treat sample and comparison stimuli as separate cues rather than configuring them into unitary stimuli.

At the end of the delay interval, the houselight was illuminated and simultaneously the comparison acoustic stimulus was presented by the speaker under the left lever. Responses on the left lever were not effective during the .8-sec enforced listening period for comparison stimuli. The comparison stimulus continued until the subject pressed the left lever or until an additional $3 \mathrm{sec}$ (the "response window") had elapsed.

On trials in which the comparison stimulus was the same as the sample (matching trials), a correct response was defined as a press of the left lever during the $3-\mathrm{sec}$ response window. Such correct responses resulted in termination of the comparison stimulus, delivery of a food pellet, and entry into the intertrial interval (ITI). Failure to respond within the 3-sec window was an incorrect response and resulted in a time-out (usually $60 \mathrm{sec}$ ), during which the houselight was dimmed.

On nonmatching trials, the withholding of a response on the left lever during the 3 -sec response window was considered a correct response; such correct responses resulted in entry into the ITI but did not produce a food pellet. A press on the left lever during the 3-sec window constituted an error and led to the same time-out as on incorrect matching trials. Response latencies on the left lever were measured in units of $20 \mathrm{msec}$; a latency of $3 \mathrm{sec}$ was assigned on trials in which the left lever was not pressed during the 3 -sec window.

As already noted, the sample set in the initial matching task was gliss (G) and the low-frequency tone (LT). However, in the first two phases of training, only three sample/comparison stimulus trial types were used, one matching and two nonmatching. Pilot data had raised the concern that, when begun with all four trial types, the matching task might prove to be difficult enough to result in serious loss of attention to the auditory stimuli. It should be noted that Stepien and Cordeau (1960) also used only three trial types in the early stages of their study.

In Phase 1 , the matching and nonmatching sample/comparison stimulus pairs were $G / G, G / L T$, and $L T / G$, half of the daily trials being of the first type. Phase 2 differed from Phase 1 in substituting $\mathrm{LT} / \mathrm{LT}$ for $\mathrm{G} / \mathrm{G}$. In the terminal Phase 3 , there were equal numbers of trials with all four trial types. To assist the animals in coping with this phase. they were cycled through Phases 1 and 2 twice before being exposed to Phase 3 .

Note that, in Phases 1 and 2, the animals did not need to compare sample and comparison stimulus in order to achieve a moderately high level of performance. In Phase 1, for example, a $75 \%$ accuracy level would be achieved if a subject learned to always respond to the left lever when gliss was the comparison stimulus and never respond when it was LT.

To advance from one phase to the next. the subjects were required to achieve a criterial level of accuracy on each trial type: this typically was two sessions with at least 20 correct on the 24 matching trials (e.g., G/G), 10 of 12 correct on the nonmatching trial type in which the comparison stimulus was different from that of the matching trial $(\mathrm{G} / \mathrm{LT})$, and 9 of 12 correct on the other nonmatch- 
ing trial type. The criterion for completion of Phase 3 was two consecutive sessions with performance levels of at least $90 \%$ correct responses. The monkeys that completed Phase 3 were tested with 1 - and 3-sec delay intervals until they regained the Phase 3 criterion. All sessions consisted of 48 trials, with the different trial types intermixed in a quasi-random order.

The main departures from the above procedure were as follows. Moe and Boris, the lead animals in the study, were initially trained with all four trial types, but neither showed any sign of learning the matching task after some 2,000 trials. They were then shifted to the three-trial-type procedure. Boris received about 1,800 trials with a symmetrical reinforcement procedure, in which a food pellet was given on correct nonmatching trials, before starting the standard Phase 1 training. Tiny, one of the last subjects run in the study, was presented in Phase 1 with the two matching trial types and one nonmatching type (G/LT); in Phase 2, LT/G was substituted for G/LT. He was moved to Phase 3 after one, rather than two, cycles on Phases 1 and 2, the criterion for advancement being $85 \%$ correct responses during a single session.

Transfer tests. The monkeys that acquired the first matching task were given five transfer tests, each with a different pair of acoustic stimuli, which will be described in conjunction with the obtained results. Tests 1 and 2 were conducted with a 1 -sec delay interval separating sample and comparison stimulus; in Tests 3-5, the delay interval was $.5 \mathrm{sec}$. Each test continued until the subject reached the criterion of two consecutive sessions with at least $90 \%$ correct responses. After the subjects reached criterion on Tests $1-4$, the delay interval on half of the trials was increased to $3 \mathrm{sec}$, and the animals were continued on the task until the previous criterion was again met.

\section{RESULTS}

\section{Acquisition of Matching}

Four of the 8 subjects, Boris, Moe, Tiny, and Tom, learned the initial matching task, requiring a total of 1,152 to 3,360 trials to complete Phases 1-3 (Table 1).

Of the four monkeys that failed to learn, three (Dag- wood, Fifi, and Phurp), were unable to meet the Phase 1 criteria within their allotted trials. Table 1 reveals that Dagwood and Fifi did not complete the first cycle of Phase 1 despite 2,400 training trials. In the hope that a new sample/comparison stimulus pair would increase their attention to the acoustic stimuli, they were advanced to Phase 2. When they failed to satisfy the performance criteria within an additional 2,400 trials, they were discontinued from the experiment. Phurp was unable to complete Phase 1 within the allotted 2,880 trials. Because he was performing very poorly at the end of the first 1,400 trials, we changed the trial types from one matching and two nonmatching to two matching and one nonmatching, the same as those employed with Tiny. This change did not improve his performance. Although Atom required 2,736 trials, he managed to complete the first cycle of Phase 1. However, after 1,440 trials on Phase 2, he was performing at a chance level. He was returned to Phase 1, employing the two-matching-trials condition, and when he failed to meet the accuracy criteria within 1,440 trials, he was removed from the experiment.

In Table 1 the mean percentages of correct responses during the last two sessions of Phases 1 and 2 are shown in parentheses. When terminated from the study, three of the above four subjects were responding at chance levels. In view of his poor performance in Phase 1, Atom's terminal performance in the second cycle of Phase $1(75 \%)$ is probably based on the discriminative process described earlier rather than on matching. For comparison purposes, the corresponding percentages are presented for Boris, Moe, and Tom, which were subjected to the same performance criteria during Phases 1 and 2 as the 4 monkeys that failed to learn the matching task.

Table 1

Results From the Initial Auditory Matching-to-Sample Task

\begin{tabular}{|c|c|c|c|c|c|c|c|}
\hline \multirow[b]{2}{*}{ Subject } & \multirow[b]{2}{*}{$\begin{array}{c}\text { Age } \\
\text { (Years) }\end{array}$} & \multirow[b]{2}{*}{ Sex } & \multirow[b]{2}{*}{$\begin{array}{c}\text { Delay } \\
\text { (Seconds) }\end{array}$} & \multicolumn{4}{|c|}{$\begin{array}{l}\text { Trials Received, and Percentage Correct () } \\
\text { Over Last Two Sessions of the Phase }\end{array}$} \\
\hline & & & & $\begin{array}{c}\text { Phase } 1 \\
\text { (Cycles 1 \& 2) }\end{array}$ & $\begin{array}{c}\text { Phase } 2 \\
\text { (Cycles } 1 \& 2 \text { ) }\end{array}$ & Phase 3 & $\begin{array}{l}\text { Total } \\
\text { Trials }\end{array}$ \\
\hline Boris & 6 & $\mathbf{M}$ & 1.0 & $\begin{array}{l}288(95) \\
288(93)\end{array}$ & $\begin{array}{r}1296(92) \\
336(97)\end{array}$ & 192 & 2,400 \\
\hline Moe & 4 & $\mathbf{M}$ & 1.0 & $\begin{array}{l}816(87) \\
288(90)\end{array}$ & $\begin{array}{r}864(97) \\
96(96)\end{array}$ & 624 & 2,688 \\
\hline Tom & 4 & M & .5 & $\begin{array}{r}1104(89) \\
672(93)\end{array}$ & $\begin{array}{l}960(92) \\
432(96)\end{array}$ & 192 & 3,360 \\
\hline Tiny & 4 & $\mathbf{M}$ & .5 & $\begin{array}{l}432 \\
\text { NR }\end{array}$ & $\begin{array}{r}96 \\
\text { NR }\end{array}$ & 624 & 1,152 \\
\hline Atom & 5 & $\mathbf{M}$ & .5 & $\begin{array}{r}2,736(83) \\
* 1,440(75)\end{array}$ & $\begin{array}{l}* 1,440(53) \\
\text { NR }\end{array}$ & NR & 5,616 \\
\hline Dagwood & 22 & $F$ & 1.0 & $\begin{array}{l}* 2,400(68) \\
\quad \mathrm{NR}\end{array}$ & $\begin{array}{l}* 2,400(54) \\
\quad \mathrm{NR}\end{array}$ & NR & 4,800 \\
\hline Fifi & 21 & $\mathrm{~F}$ & 1.0 & $\begin{array}{l}* 2,400(78) \\
\quad N R\end{array}$ & $\begin{array}{l}* 2,400(55) \\
\quad N R\end{array}$ & NR & 4.800 \\
\hline Phurp & 14 & $\mathbf{M}$ & .5 & $\begin{array}{l}* 2,880(49) \\
\text { NR }\end{array}$ & $\begin{array}{l}\text { NR } \\
\text { NR } \\
\end{array}$ & NR & 2,880 \\
\hline
\end{tabular}

Note $-N R=$ Subject was not run in this phase and cycle. *Subject did not satisfy performance criteria in this phase and cycle. 
In most cases, the terminal performances achieved in the two phases and cycles were $90 \%$ or higher.

Tiny's rapid acquisition of the matching task indicates that two cycles of Phases 1 and 2, although probably helpful, are not necessary for successful auditory matching. As pointed out below, however, Tiny had had much more previous experience with acoustic stimuli than any of the other subjects, so two cycles may be generally advisable. It is clear from Table 1 that the duration of the delay interval, .5 or $1.0 \mathrm{sec}$, was not an important factor.

\section{Transfer Tests}

Two dependent variables were available for analysis, percentage of correct responses (correct and incorrect responses as defined above) and response latencies on matching and nonmatching trials (which were subjected to Mann-Whitney $\mathrm{U}$ tests). Because it is a dichotomous variable, percentage of correct responses is likely to be somewhat less sensitive than response latency, a continuous variable within its range of permissible values. This was borne out by analyses of the transfer data conducted with both dependent variables. Although the outcomes were essentially the same with both measures, differences in response latencies sometimes yielded slightly more significant results. Consequently, only the analyses based on percentages of correct responses are reported.

Figure 1 presents the results of Transfer Tests 1 and 2. In Test 1, the high-frequency tone was substituted for the gliss tune. As a result, three of the four sample/comparison stimulus pairs were new: HT/HT, HT/LT, and LT/HT. The isolated points to the left of the connected lines give the percentages of correct responses during the first 24 trials of the initial test session. Three of the 4 subjects performed significantly better than chance on these trials. (For 24 trials, a performance level of $75 \%$ correct is significant at the .03 level by a two-tailed binomial test.) The horizontal dotted lines in the figure indicate the twotailed binomial .01 and .001 levels of significance for percentage of correct responses based on all 48 trials of a session. All 4 subjects showed a high degree of transfer to the Test 1 stimuli, Boris reaching the standard test criterion of two consecutive sessions with at least $90 \%$ correct responses in only four sessions, including the two criterial sessions.

In Test 2, gliss was substituted for LT, so that only two sample/comparison stimulus pairs were new, G/HT and $\mathrm{HT} / \mathrm{G}$. Boris and Tom transferred immediately and virtually completely to the auditory stimuli of Test 2 ; they performed at a very high level on the first 24 test trials and met criterion in two sessions, the smallest number possible. Although less impresssive, Moe and Tiny performed well above chance on the first 24 trials and on all of the individual sessions.

The results of Transfer Tests 3-5 are presented in Figure 2. One stimulus of Test 3 was the (steady) medium tone (MT); the other was the previously used HT and LT, the two tones alternating every $.7 \mathrm{sec}$. Inasmuch as the animals had never matched either of these stimuli, all four sample/comparison stimulus pairs may be said to be new. Figure 2 shows that all 4 subjects transferred to the new acoustic stimuli at a very high level; indeed, three of the monkeys reached criterion in the minimum of two sessions, and Moe required only three.

The Test 3 stimuli differed not only in their frequency components, but in their tempo as well. For the stimuli of Test 4 , the tempo difference was eliminated by imposing the same pulse rate on both. The MT was pulsed at a rate of $4 \mathrm{~Hz}$, with approximately equal on and off times.

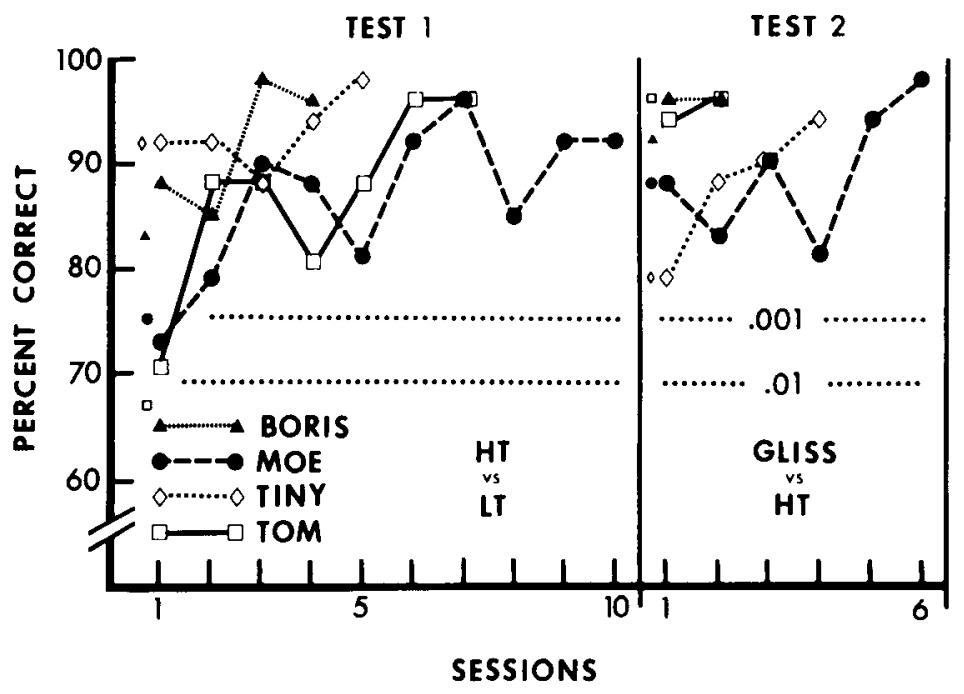

Figure 1. Percentages of correct responses on each 48 -trial session of Tests 1 and 2 . The isolated points to the left of the connected lines give the percentages correct for the first 24 trials of the initial test session. Significance levels for the 48-trial sessions are shown by the dotted lines (see text). The acoustic stimuli of Test 1 were a high-frequency tone (HT) and a low-frequency tone (LT). In Test 2, they were a gliss "tune" and HT. 


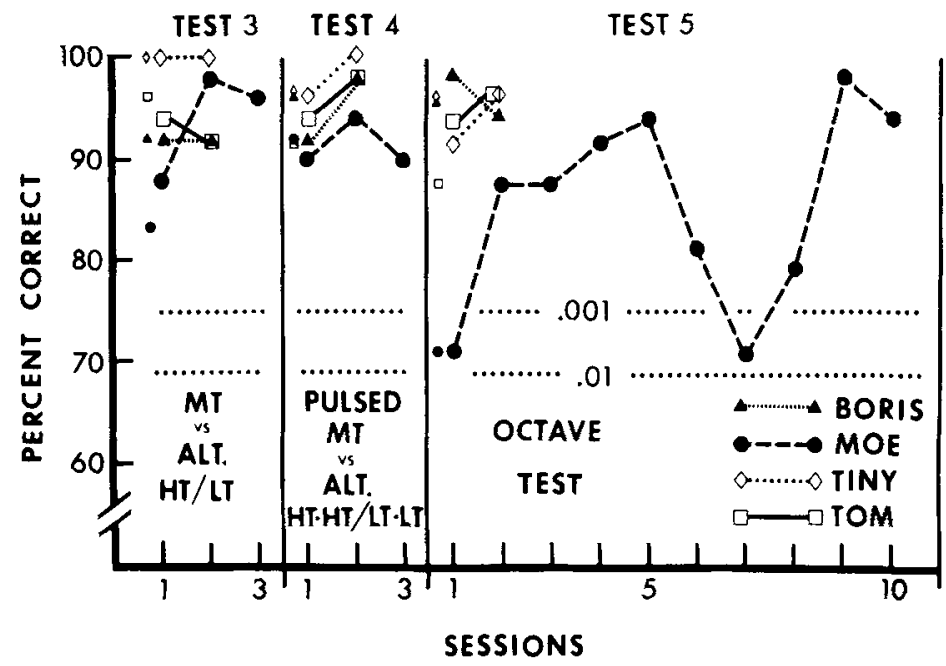

Figure 2. Percentages of correct responses on each 48-trial session of Tests 3-5. The isolated points to the left of the connected lines give the percentages correct for the first $\mathbf{2 4}$ trials of the initial test session. Significance levels for the 48 -trial sessions are shown by the dotted lines (see text). The acoustic stimuli of Test 3 were a medium-frequency tone (MT) and the HT and LT alternating every .7 sec (ALT.HT/LT). The tempo difference between the stimuli used in Test 3 was eliminated in Test 4 by pulsing each stimulus at the same rate (see text). Test 5 employed the same acoustic stimuli as Test 4 , raised by 1 octave.

The alternating HT/LT stimulus was pulsed at the same rate and with the same on/off times, but the train of pulses consisted of alternating pairs of the two tones, that is, HT.HT/LT.LT. Figure 2 reveals that 4 subjects showed an extremely high level of transfer to these stimuli. Again, only Moe required more than the minimum of two sessions to reach the performance criterion.

In an earlier study, we found that monkeys highly trained on a tune discrimination failed to transfer the discrimination when the tunes were raised or lowered by an octave (D'Amato \& Salmon, 1984a). A similar result has been reported with birds (Hulse \& Cynx, 1985), suggesting that, despite an early positive report on the performance of rats (Blackwell \& Schlosberg, 1943), octave generalization may not be a robust phenomenon in animals (but see Richards, Wolz, \& Herman, 1984). In the final transfer test, the ability of the monkeys to match stimuli that were transformed by 1 octave was assessed.

The stimuli of Test 5 were those of Test 4 raised 1 octave (all frequencies doubled). As shown in Figure 2, three subjects responded to the transformed stimuli at a very high level of accuracy. Moe performed poorly on the first test session and was untypically erratic in reaching criterion, suggesting a motivational problem. In any case, if the octave transformation altered the recognizability of the Test 4 stimuli, most of the monkeys had no difficulty in applying the matching rule to the new stimuli.

\section{Tests with 3-sec Delays}

After meeting the criterion of at least $90 \%$ correct in two consecutive sessions on the initial matching task and on Transfer Tests 1-4, the delay interval on half of the 48 trials was increased to $3 \mathrm{sec}$ and the subjects were re- quired to satisfy the same criterion a second time. Figure 3 presents the number of sessions needed by each subject to regain criterion with the new delay conditions. Apart from Moe on Tests 3 and 4, the animals had little difficulty with the 3-sec delay interval, in many cases reaching criterion in the minimum of two sessions.

\section{DISCUSSION}

The acquisition results of the present study demonstrate that, with appropriate training procedures, cebus monkeys can learn auditory matching-to-sample to a high level of proficiency. Very recently, auditory matching was reported in Japanese macaque monkeys (Macaca fuscata), also in a go/no-go paradigm (Kojima, 1985). The two monkeys in that study were first trained on discrimination and discrimination reversal of the two acoustic stimuli later to be matched and then required an additional $3,000-4,000$ matching trials to reach an unspecified acquisition criterion, which, judging from their performance at a 2-sec delay, was less stringent than that employed in the present study. This, plus the difficulty encountered when the delay interval was increased beyond the training value of $.5 \mathrm{sec}$, suggests that Kojima's training procedures may not have been as efficient as those employed in the present study.

The results obtained from the five transfer tests to new acoustic stimuli are significant in that they represent, to the best of our knowledge, the first clear evidence of the matching concept in the auditory modality of monkeys. During acquisition of the initial matching task, the animals must have learned something more general than associating a specific response (press, or refrain from pressing, 


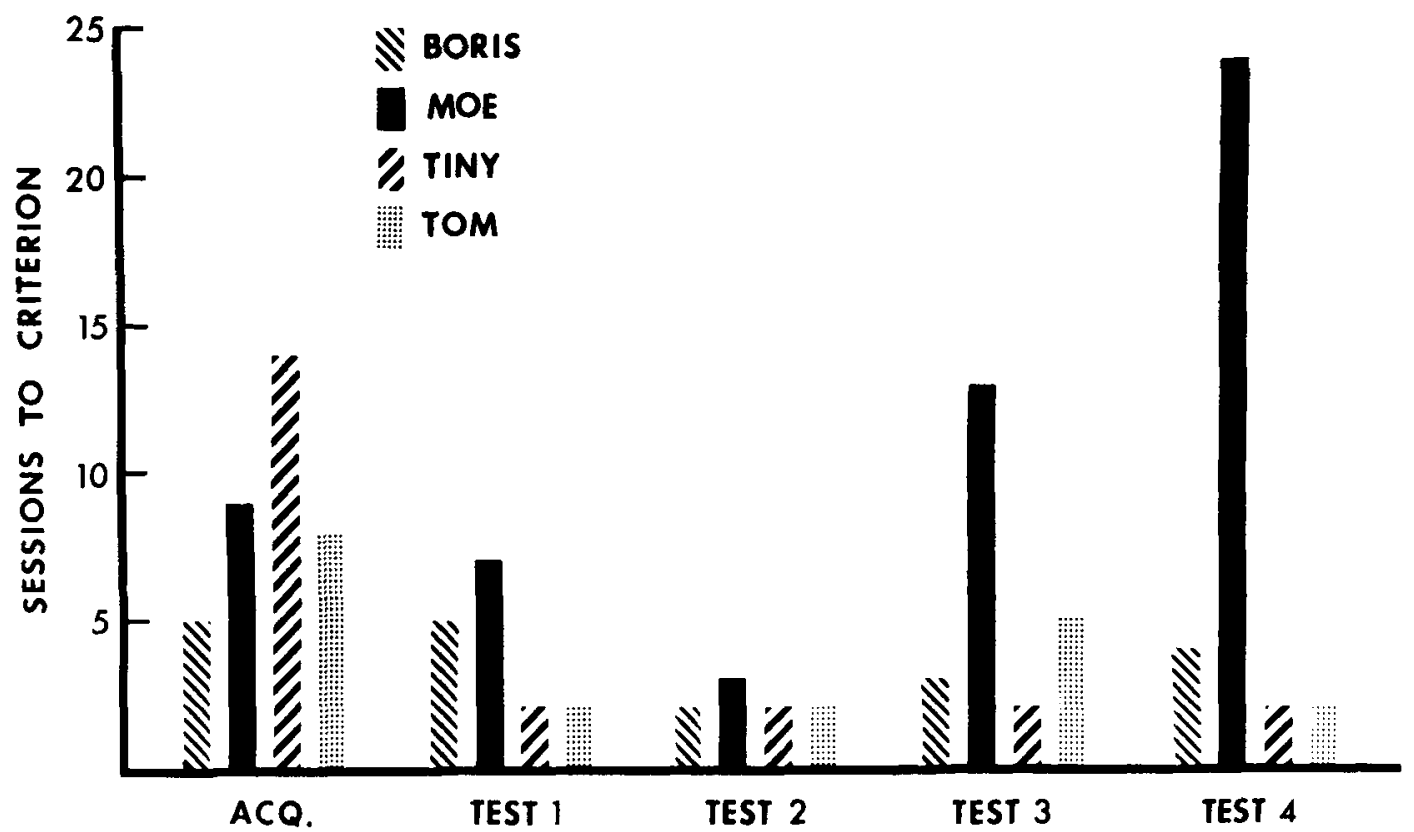

Figure 3. The numbers of sessions required to regain criterion after increasing the delay interval on 24 of the 48 trials from .5 or $1 \mathrm{sec}$ to $3 \mathrm{sec}$. The 3-sec delay interval was added after criterion was achieved with the shorter delay in acquisition of the initial matching task (ACQ.) and Tests 1-4.

the left lever) to each of the four sample/comparison stimulus pairs. It is difficult to account for their performances on the first 24 trials of Transfer Test 1 on that basis. Similarly, the high performance levels achieved by most of the subjects during the initial 24 trials of Tests 2-5 and the rapidity with which criterion was often achieved in these tests strongly implicate concept-mediated transfer. Indeed, a high degree of transfer was evident even in the first 12 trials of each of the five transfer tests. The group mean percentages of correct responses over the first 12 trials were $79.2,83.3,93.8,93.8$, and 89.6 for Tests 1-5, respectively; of the 20 individual-subject percentages available for the initial 12 transfer trials, 17 were $83.3 \%$ or higher.

The 3-sec delay intervals that were introduced after the subjects met criterion in acquisition and in Tests $1-4$ had the joint purposes of assessing the monkeys' retention of the sample stimuli and providing an additional means of assessing whether their performances were based on matching or on associating specific responses to each sample/comparison stimulus configuration. We hypothesized that if it was the latter, the introduction of the 3-sec delay intervals might significantly impair matching accuracy, because a sample/comparison stimulus perceptual configuration established with a .5- or 1-sec delay could be disrupted by a sudden increase of the delay to $3 \mathrm{sec}$. That the 3-sec delays introduced in Tests $1-4$ had little effect on performance (in the case of three of the subjects, at least) reveals good retention of the sample stimulus over this interval and is consistent with the conclusion that the monkeys were matching the comparison stimulus to the sample.
Moe may be an exception. He required more sessions to reach criterion with the 3-sec delays in Tests 1-4 than any of the other animals, and the same was true with regard to his achieving criterion in Tests $1-5$. On the other hand, his performance on the initial 24 trials of Tests 1-4 was significantly above chance levels.

Inasmuch as 4 of the 8 subjects failed to learn the initial matching task, it is clear that favorable training conditions are not sufficient by themselves to insure successful auditory matching-to-sample. Table 1 shows that 3 of the 4 unsuccessful monkeys were considerably older than the 4 successful subjects. Therefore, age might be a factor. Atom's age was within the range of the latter monkeys, and it may be significant that he was the only one of the unsuccessful animals to reach criterion in Phase 1.

Previous experimental experience with visual and acoustic stimuli is probably a more important factor than age. Dagwood and Fifi had had an enormous amount of previous training with visual stimuli, most with matchingto-sample. In contrast, Dagwood had encountered auditory discriminative stimuli in only one study (D'Amato \& Salmon, 1982), and Fifi had no such previous training at all. Phurp also had had very extensive previous visual matching-to-sample training; on the other hand, his training with acoustic stimuli was limited to auditory-visual conditional matching (D'Amato \& Salmon, 1984b). Among the unsuccessful subjects, Atom had had the greatest amount of past training with auditory stimuli, although it was less than the training he had received with visual discriminanda.

Turning to the four successful monkeys, in all cases their first experimental training had been with acoustic 
stimuli in an operant discrimination task (D'Amato \& Salmon, 1984a). Boris, Moe, and Tom received between 1,300 and 1,550 training-test trials in that study, and Tiny, the fastest learner in the present experiment, had the benefit of almost 12,000 trials. Before the present experiment was begun, Tom had received about 5,000 trials on an auditory-visual conditional matching task, and Boris, Moe, and Tiny had been exposed to approximately 5,500, 9,300 , and 9,900 visual matching trials, respectively (D'Amato et al., 1985). It is interesting that Moe, the poorest performer of the successful monkeys, had had about twice as much previous experience with visual discriminative cues as Boris or Tom. Tiny's very extensive prior experience with acoustic stimuli may have been an important factor in sparing him the negative effects of his subsequent training on visual matching, which was somewhat more than that received by Moe.

Although only correlational, the available evidence implicates the relative amounts of past experience with visual and auditory discriminanda as a factor in the degree to which acoustic stimuli are capable of gaining stimulus control. It may not be an accident that Stepien and Cordeau's (1960) monkeys were experimentally naive. However, Thompson's (1980) monkeys were also experimentally naive, but it is possible that his training procedures were not as favorable for establishing auditory matching in monkeys as Stepien and Cordeau's, which were not radically different from our own. (The previous experimental experience of Kojima's monkeys was not reported.)

One might have supposed that the vast amounts of visual matching-to-sample training received by some of the unsuccessful monkeys would have facilitated auditory identity matching, inasmuch as the matching rule would have been thereby well entrenched. However, we have shown that within the visual modality itself the matching concept tends to have limited generality, being restricted to the class of visual stimuli with which it was developed (D'Amato et al., 1985). Monkeys trained to match two forms or a form and a red disk ("static" visual stimuli) showed concept-mediated transfer when tested with new static stimuli. However, when later tested with a steady versus flashing green disk ("dynamic" visual stimuli), they gave no evidence of applying the matching concept, and indeed it appeared that they had to reacquire the concept for this new class of stimuli. Given the limited abstractness of the matching concept within the visual modality, it is perhaps not surprising that extensive matching-to-sample experience in the visual modality does not facilitate auditory matching.

Returning to the theoretical issue that motivated the present study, the evidence for concept-mediated transfer produced by Tests $1-5$ does not support the hypothesis that complex cognitive processes are modality specific in animals, at least not the strong form of that hypothesis. Under appropriate conditions, monkeys can learn to match acoustic stimuli and apply the matching rule to novel stimuli. However, monkeys do seem to acquire matching more readily, and with fewer constraints, in the visual modality. For example, in spite of their previous experience with acoustic stimuli (very extensive in the case of Tiny), Boris and Tiny subsequently learned a visual matching task readily and gave strong indications of concept-mediated transfer to a new set of visual stimuli (D'Amato et al., 1985). It seems unlikely that they would have performed as well on an auditory matching task had it been preceded by a like amount of training with visual stimuli. The preferential status of the visual modality with regard to the expression of complex learning and cognitive functions in monkeys is perhaps better described as modality asymmetry rather than modality specificity.

Finally, why is this modality asymmetry accentuated by previous training with visual stimuli within the same experimental context? Drastic "tuning out" of the acoustic stimuli does not appear to be the answer. The unsuccessful monkeys seemed to attend readily enough to the onset of the sample stimuli, as judged by their latencies to press the right lever. And they showed some degree of sensitivity to the comparison stimuli, in that they were more likely, in Phase 1 (in which $\mathrm{G} / \mathrm{G}$ was the matching trial type), to press the left lever on a LT/G nonmatching trial than to do so on the G/LT type. What seemed lacking was sufficiently sustained attention to the sample/comparison stimulus sequence to detect the relationship between the two acoustic components. Apparently, the sample was used primarily as a cue to press the right lever, which advanced them to the comparison stimulus stage. They were then disposed to respond to the comparison stimuli on the basis of a simple $S+/ S-$ task.

The reasons why this strategy, which to some degree was adopted by all 8 monkeys, persisted so long in the unsuccessful subjects (whether or not they had had previous experience with an auditory $\mathbf{S}+/ \mathbf{S}-$ discrimination) are not at all clear. Extensive past experience with visual discriminanda in the same setting may strongly pretune the animals to attend to visual cues, whether relevant or not. In the present study, for example, the houselight was turned off during the short delay that separated sample and comparison stimulus (the aim of this was to discourage the monkeys from configuring the two cues into a unitary stimulus). This change in the conditions of illumination could have so commanded the animal's attention to the visual modality as to interrupt all processing of the acoustic sample, which obviously would have impeded relating a trial outcome to the nature of the sample/comparison stimulus sequence. Whatever the mechanisms by which extensive past training with visual discriminanda inhibits the development of auditory matching in monkeys, its effects are often powerful and long-lasting.

\section{REFERENCES}

BlaCKWELl, H. R. \& SCHLOSBERG, H. (1943). Octave generalization, pitch discrimination, and loudness thresholds in the white rat. Journal of Experimental Pscyhology, 33, 407-419.

Cowey. A. (1968). Discrimination. In L. Weiskrantz (Ed.), Analysis of behavioral change (pp. 189-238). New York: Harper \& Row. DAmato. M. R. \& Colombo, M. (1985). The matching concept in 
monkeys. In G. d'Ydewalle (Ed.), Cognition, information processing, and motivation (Proceedings of the XXIII International Congress of Psychology, Vol. 3, pp. 19-36). Amsterdam: Elsevier Science Publishers.

D'Amato, M. R., \& Salmon, D. P. (1982). Tune discrimination in monkeys (Cebus apella) and in rats. Animal Learning \& Behavior, 10, 126-134.

D'amato, M. R., \& Salmon, D. P. (1984a). Processing of complex auditory stimuli (tunes) by rats and monkeys (Cebus apella). Animal Learning \& Behavior, 12, 184-194.

D'Amato, M. R., \& Salmon, D. P. (1984b). Processing and retention of complex auditory stimuli in monkeys (Cebus apella). Canadian Journal of Psychology, 38, 237-255.

D'Amato, M. R., Salmon, D. P., \& Colombo, M. (1985). Extent and limits of the matching concept in monkeys (Cebus apella). Journal of Experimental Psychology: Animal Behavior Processes, 11, 35-51.

Herman, L. M., Richards, D. G., \& Wolz, J. P. (1984). Comprehension of sentences by bottlenosed dolphins. Cognition, 16, 129-219.

HuLSE, S. H., \& CYNX, J. (1985). Relative pitch perception is constrained by absolute pitch in songbirds (Mimus, Molothrus, and Sturnus). Journal of Comparative Psychology, 2, 176-196.

KoJima, S. (1985). Auditory short-term memory in the Japanese monkey. International Joumal of Neuroscience. 25, 255-262.

Richards, D. G., Wolz, J. P., \& Herman, L. M. (1984). Vocal mimicry of computer-generated sounds and vocal labeling of objects by a bottlenosed dolphin, Tursiops truncatus. Journal of Comparative Psychology, 98, 10-28.

Salmon, D. P. (1984). An investigation of modality specificity in the cognitive processes of monkeys (Cebus apella) (Doctoral dissertation, Rutgers-The State University, 1984). Dissertation Abstracts International, 45, 706B.

Segal, M., \& Harrison, J. M. (1978). The control of responding by auditory stimuli: Interactions between different dimensions of the stimuli. Joumal of the Experimental Analysis of Behavior, 30, 97-106.

Stepien, L. S., \& Cordeau, J. P. (1960). Memory in monkeys for compound stimuli. American Journal of Psychology, 73, 388-395.

Thомpson, R. K. R. (1980, July). Auditory cued reversal and matchingto-sample learning by rhesus monkeys. Paper presented at the Eighth International Congress of Primatology, Florence, Italy.

TномрSоN, R. K. R. (1981, October). Nonconceptual auditory matching by a rhesus monkey reflects biological constraints on cognitive processes? Paper presented at the Northeastern Meeting of the Animal Behavior Society, Kingston, Ontario, Canada.

WEGENER, J. G. (1964). Auditory discrimination behavior of normal monkeys. Journal of Auditory Research, 4, 81-106.

(Manuscript received June 24, 1985;

Revision accepted for publication October 18, 1985.) 